\title{
Responsiveness and reliability of the Sinus Control Test in chronic rhinosinusitis*
}

\author{
Preeti Kohli', Zachary M. Soler', Kristina A. Storck', Arash Shahangian', \\ Sarfaraz M. Banglawala, ${ }^{3,4}$ Rodney J. Schlosser ${ }^{1,2}$ \\ 'Division of Rhinology and Sinus Surgery, Department of Otolaryngology - Head and Neck Surgery, Medical University of South \\ Carolina, Charleston, SC, USA \\ 2 Department of Surgery, Ralph H. Johnson VA Medical Center, Charleston, SC, U.S.A \\ ${ }^{3}$ Department of Otolaryngology-Head and Neck Surgery, University of Toronto, Toronto, ON, Canada \\ ${ }^{4}$ Department of Surgery, Otolaryngology-Head and Neck Surgery Division, McMaster University, Hamilton, ON, Canada
}

Rhinology 55: 39-44, 2017

https://doi.org/10.4193/Rhino16.208

*Received for publication:

July 4, 2016

Accepted: August 23, 2016

\begin{abstract}
Background: The Sinus Control Test (SCT) is a patient-reported questionnaire designed to help physicians identify sub-optimally controlled chronic rhinosinusitis (CRS). This study measures responsiveness to surgery and reliability of the SCT.

Methodology: Adults meeting diagnostic criteria for CRS were recruited from rhinology clinics at a tertiary academic institution. To measure responsiveness, the SCT was administered at baseline and at least 3 months after surgery to 62 CRS patients. To measure reliability, the SCT was administered at two clinical encounters a maximum of 14 days apart to 22 CRS patients.

Results: Total SCT scores significantly improved from baseline to post-operative follow-up, and the distribution of patients with total SCT scores falling into the "uncontrolled," "partially controlled," and "controlled" categories before and after surgery were significantly different in the direction of improvement. The SCT met minimum standards for reliability and internal consistency as measured by: test-retest reliability coefficient, intra-class correlation coefficients, and item-total correlations. Cronbach's a values with each item deleted were lower than the overall Cronbach's a. The SCT captures the full range of disease control as measured by floor and ceiling effects.
\end{abstract}

Conclusion: The SCT is responsive to surgical intervention and a reliable tool to monitor changes in CRS control levels.

Key words: Patient-reported metrics, sinusitis, disease control, validation, endoscopic sinus surgery

\section{Introduction}

Chronic rhinosinusitis (CRS) is a common disease of the sinonasal mucosa characterized by persistent inflammation. Long-term management of CRS includes treating repeat exacerbations and infections, which significantly impact morbidity. Through available treatments, the disease can often be managed, but patients fluctuate between various degrees of disease control ${ }^{(1)}$. Clinical outcomes in CRS often revolve around optimizing patient symptoms and quality of life (QOL); however, measures of disease control provide complementary information in understanding such clinical outcomes. For example, patient symptoms may be minimized, but if this requires prolonged courses of oral antibiotics and oral steroids, then CRS may not be truly well controlled despite outstanding QOL scores. The goal of CRS treatment is to maintain clinical control, defined as "a disease state in which the patients do[es] not have symptoms or the symptoms are not bothersome" and only the need for local medication (2)". Correctly identifying when a patient is no longer controlled is necessary to facilitate the additional treatment or proper referrals required to maintain a stable disease state. Control instruments are widely used in managing other airway disorders, such as asthma and rhinitis. Surveys have found that physicians and patients tend to overestimate asthma control, leaving a significant portion of patients with uncontrolled disease and tolerating manageable symptoms ${ }^{(3)}$. As a result, guidelines from the National Asthma Education and Prevention Program (NAEPP) advocate the use of asthma control instruments to better monitor variable disease course ${ }^{(3,4)}$. These guidelines also make the distinction between quality of life instruments, which evaluate patient perception of disease impact, and 
control instruments, which guide decisions around adjusting therapy and evaluating treatment response ${ }^{(3,4)}$. For example, in asthma, patients who feel their lives are minimally impacted while frequently using rescue medications are at risk for future exacerbations, hospitalization, and intubation, which should be addressed by a physician. Therefore, control and quality of life instruments serve complementary but separate purposes in patient care.

Chronic rhinosinusitis is also a highly variable disease, which requires a similar level of monitoring. The Sinus Control Test (SCT) was developed by Banglawala et al. to provide physicians with a brief, patient-reported tool to evaluate disease control in a clinical setting ${ }^{(5)}$. The SCT is a 4-item questionnaire that evaluates severity of nasal obstruction and nasal discharge as well as productivity loss and medication use. Scores range from 0-16 and categorize patients as "controlled," "partially controlled," and "uncontrolled." Items in the SCT were selected based on systematic review, focus groups of CRS patients, and input from multi-disciplinary (rhinology, allergy, pediatrics, and primary care) experts (5). In an initial paper, the SCT met validity criteria and was shown to correlate with standard disease measurements such as the Sinonasal Outcome Test (SNOT-22), Lund-Kennedy endoscopy score, and physician assessment. In a recent review on the quality of patient-reported outcome metrics (PROMs) in CRS, the SCT fulfilled criteria for reporting of developmental properties; however, several psychometric properties are yet to be reported ${ }^{(6)}$. The main objective of this study was to examine the responsiveness of the SCT to surgical intervention. The secondary aims were to examine test-retest reliability, internal consistency, and floor/ceiling effects, as well as to explore factors impacting change in SCT scores.

\section{Materials and methods}

\section{Study population}

Adults ( $\geq 18$ years) meeting diagnostic criteria for CRS as defined by current Clinical Practice Guideline (CPG) of the American Academy of Otolaryngology-Head and Neck Surgery (AAOHNS)

(7) and the European Position Paper on Rhinosinusitis and Nasal Polyps (EPOS2012)(2) were enrolled from rhinology clinics at the Medical University of South Carolina (MUSC). All included patients provided written informed consent and were literate in English. The Institutional Review Board at MUSC approved the study (Pro \#14124). All patients electing to undergo endoscopic sinus surgery (ESS) had persistent symptoms despite initial medical management consisting of at least one course of oral, broad spectrum or culture- directed antibiotics for at least 14 days with either a minimum 3 weeks of topical steroid sprays or 5 days of systemic corticosteroid therapy. Extent of surgical treatment was left to the surgeon's discretion.

Demographic information and history of co-morbidities was collected by patient-self report and medical chart review. Baseline computed tomography (CT) scan was scored using the LundMackay staging system ${ }^{\left({ }^{8}\right)}$. Nasal endoscopy was obtained and scored using the Lund-Kennedy staging system at each clinic visit ${ }^{(9)}$.

\section{Responsiveness}

Responsiveness is the ability of a PROM to detect clinically significant change over time and was assessed by comparing SCT scores before and after ESS ${ }^{(6)}$. Patients completed the Sino-Nasal Outcome Test (SNOT-22), consisting of 22-symptom related items on a 0-5 Likert scale ${ }^{(10)}$, and Sinus Control Test (SCT) at baseline and at 3-month post-operative visit or after. If patients were not able to come into physician's office for follow-up visit, SCT and SNOT-22 were collected by phone call or by electronic mail survey administered through the Research Electronic Data Capture (REDCap) database. Paired t-tests were used to compare pre- and post-surgical SCT and SNOT-22 scores. Distributions of $\mathrm{SCT}$ categories were compared using the Pearson chi-square test or Fisher's exact test.

\section{Reliability}

Test-retest reliability, which measures the stability of PROMs over time, was assessed by correlating responses to questionnaires administered at two time points between which the disease does not significantly change ${ }^{(6)}$. Patients completed the questionnaires at two separate clinical encounters, a maximum of 14 days apart, without intervening changes in treatment. Pearson's correlation coefficient was used to quantify relationship between test-retest scores, and the threshold for acceptability was set at $\geq 0.70^{(6)}$. In addition, intra-class correlation coefficients (ICC) were used to measure the score reproducibility of each individual question. Threshold for acceptability was defined as $0.4-0.8$, with scores $>0.8$ indicating near perfect agreement $^{(11)}$.

\section{Internal consistency}

Internal consistency, considered another measurement of reliability, indicates the degree to which separate items in a PROM measure the same underlying construct ${ }^{(6)}$. Item-total correlations, which measure the correlation between individual items and the total score, overall Cronbach's a, and Cronbach's a with each item deleted were calculated before and after surgery. Threshold for acceptability for item-total correlations and Cronbach's $a$ is 0.4 and 0.7 , respectively ${ }^{(6,12)}$.

\section{Floor and ceiling effects}

Floor and ceiling effects measure the ability of a PROM to represent the full spectrum of a construct and were assessed by determining the percentage of patients who obtained the highest or lowest possible scores ${ }^{\left({ }^{6}\right)}$. The threshold for acceptability was set at $<15 \%{ }^{(6)}$. 
Table 1. Demographic, co-morbid, and disease severity characteristics of study population.

\begin{tabular}{|c|c|c|}
\hline & Demog! & $\begin{array}{c}\text { Mean (SD) } \\
n(\%)\end{array}$ \\
\hline \multicolumn{2}{|l|}{ Age } & $50.1(15.6)$ \\
\hline \multirow{2}{*}{ Sex } & Male & $42(54.6)$ \\
\hline & Female & $35(45.5)$ \\
\hline \multirow{2}{*}{ Race } & White & $63(81.8)$ \\
\hline & African American & $14(18.2)$ \\
\hline \multirow{2}{*}{ Ethnicity } & Non-Hispanic & $75(97.3)$ \\
\hline & Unknown & $2(2.7)$ \\
\hline \multicolumn{3}{|c|}{ Co-morbidities } \\
\hline \multicolumn{2}{|c|}{ Nasal Polyposis } & $31(40.3)$ \\
\hline \multicolumn{2}{|c|}{ Allergy (by history) } & $12(15.6)$ \\
\hline \multicolumn{2}{|l|}{ Asthma } & $16(20.8)$ \\
\hline \multicolumn{2}{|l|}{ Diabetes } & $8(10.5)$ \\
\hline \multicolumn{2}{|c|}{ Depression (by history) } & $8(10.4)$ \\
\hline \multicolumn{2}{|l|}{ COPD } & $3(3.9)$ \\
\hline \multicolumn{2}{|c|}{ Fibromyalgia } & $2(2.6)$ \\
\hline \multicolumn{3}{|c|}{ Measures of Disease Severity } \\
\hline \multicolumn{2}{|c|}{ LM CT Score } & $12.2(6.2)$ \\
\hline \multicolumn{2}{|c|}{ SNOT-22 (Pre-surgical) } & $56.2(23.5)$ \\
\hline \multicolumn{2}{|c|}{ SNOT-22 (Post-surgical) } & $24.6(18.7)$ \\
\hline \multicolumn{2}{|c|}{ LK Endoscopy Score (Pre-surgical) } & $7.6(3.9)$ \\
\hline \multicolumn{2}{|c|}{ LK Endoscopy Score (Post-surgical) } & $4.0(3.1)$ \\
\hline \multicolumn{2}{|c|}{ SCT (Pre-surgical) } & $8.9(3.8)$ \\
\hline \multicolumn{2}{|c|}{ SCT (Post-surgical) } & $4.6(3.5)$ \\
\hline
\end{tabular}

COPD: Chronic Obstructive Pulmonary Disease; LM CT: Lund-Mackay Computed Tomography; SNOT-22: Sinonasal Outcome Test; LK: LundKennedy; SCT: Sinus Control Test

\section{Data storage and statistical analysis}

Data were compiled into a REDCap using double data-entry processes to ensure data integrity. All data analysis was performed using SPSS 22.0 (IBM Corporation, Armonk, NY, USA). Demographic information, co-morbidities, SNOT-22 scores, and SCT scores were assessed using descriptive statistics. Continuous variables were compared between two groups using independent-samples t-tests or Mann-Whitney U tests. Categorical variables were compared using the Pearson chi-square test or Fisher's exact test. Change was defined as the difference between pre-surgical and post-surgical SCT scores and SNOT-22 scores. To determine correlates of change in SCT after surgery, bivariate linear regression was performed with comorbidities and demographic variables. Correlates that were associated with change in SCT $(p<0.10)$ were then placed in a multiple linear regression. Variables that lost significance $(p<0.05)$ using the backward
Table 2. Differences in control status before and after surgery.

\begin{tabular}{|lccc|}
\hline & $\begin{array}{c}\text { Before Surgery } \\
\text { Frequency (\%) }\end{array}$ & $\begin{array}{c}\text { After Surgery } \\
\text { Frequency (\%) }\end{array}$ & P-value \\
\hline Controlled & $7(11.5)$ & $29(46.8)$ & \\
\hline $\begin{array}{l}\text { Partially } \\
\text { Controlled }\end{array}$ & $36(58.1)$ & $30(48.4)$ & 0.002 \\
\hline Uncontrolled & $19(30.6)$ & $3(4.8)$ & \\
\hline
\end{tabular}

selection stepwise procedure were no longer considered to be correlates of change in SCT. Resulting regression coefficients ( $\beta$ ) and standard errors (SEs) were used to estimate linear changes. Statistical significance was set at $<0.05$.

\section{Results}

\section{Study population}

There were a total of 77 patients with CRS included in the study, of which 31 were CRSwNP (40.3\%). The sample was 54.6\% male and $45.5 \%$ female with a mean age (standard deviation) of 50.1 years (15.6). Baseline demographic, co-morbid, and disease severity metrics of all study participants are shown in Table 1.

\section{Responsiveness}

Sixty-two patients completed the SCT and SNOT-22 before and after surgery. The mean duration of post-operative follow-up was $6.50 \pm 4.3$ months (range: $2.5-21$ ). Global SCT scores of patients undergoing ESS improved from $8.9 \pm 3.8$ to $4.6 \pm 3.5$ ( $p$ $<0.001$ ) (Table 1). The percentage of patients whose total SCT score fell into the "uncontrolled," "partially controlled," and "controlled" category before and after surgery was $30.6 \%$ vs $4.8 \%$, $58.1 \%$ vs $48.4 \%$, and $11.5 \%$ vs $46.8 \%$, respectively, and these distributions were significantly different $(p=0.002)$ (Table 2). Change in SCT score and change in SNOT-22 score after surgery were significantly correlated $(R p=0.480, p<0.001)$. Only age and baseline SCT score were found to be significantly associated with change in SCT score on bivariate analysis (Table 3). Importantly, the baseline SNOT-22 did not impact change in SCT after surgery. When age and baseline SCT score are both placed in the model using multiple linear regression, age becomes nonsignificant $(p=0.686)$. After adjusting for age, on average, for a one-unit increase in baseline SCT score, change in SCT increases by 0.55 (standard error: 0.10 ; t-statistic: $5.57 ; \mathrm{p}<0.001$ ).

\section{Reliability}

Twenty-two patients completed the questionnaires at two separate visits. The test-retest scores of patients were positively correlated to each other $(\mathrm{Rp}=0.782, \mathrm{p}<0.001)$. A scatter plot depicting this relationship is shown in Figure 1. The ICC for each SCT question ranged from 0.518-0.868 and are shown in Table 4. 
Table 3. Change in SCT after surgery and associations between demographics and co-morbidities.

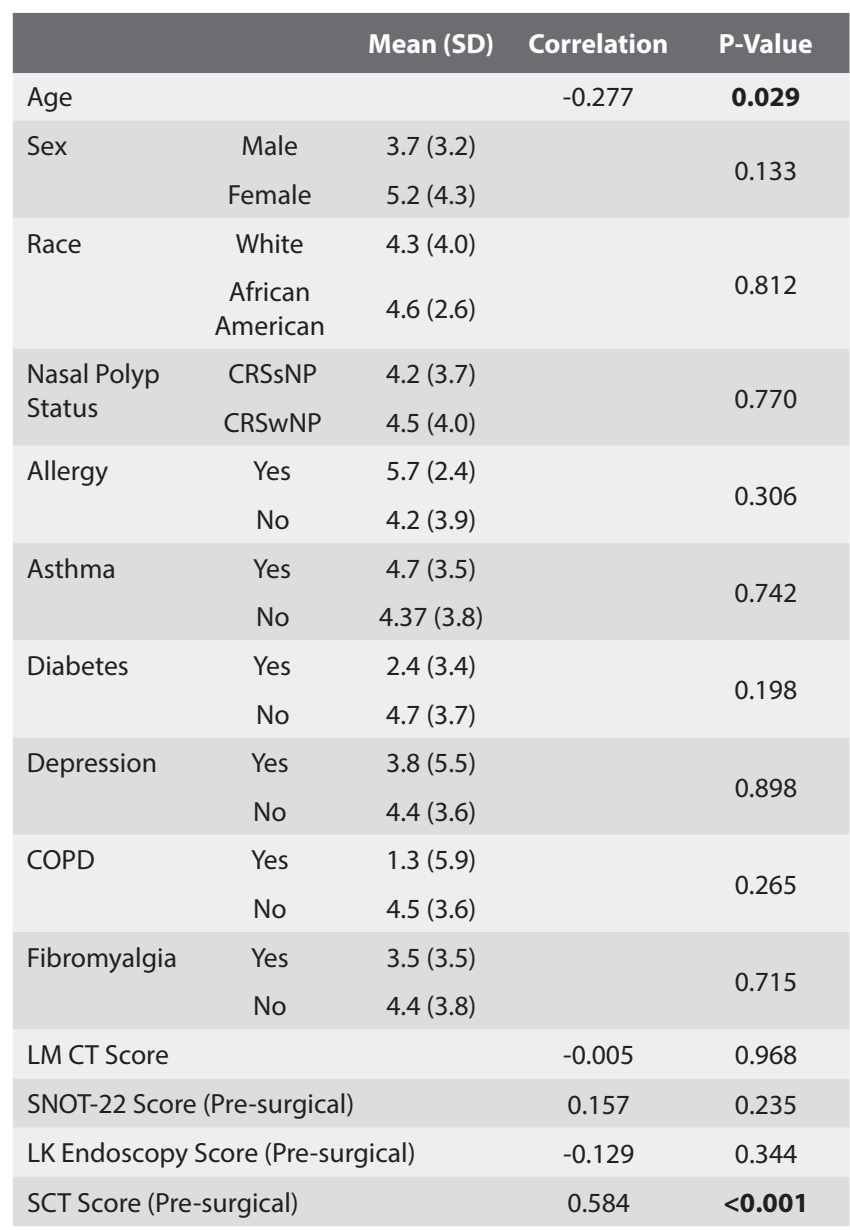

COPD: Chronic Obstructive Pulmonary Disease; LM CT: Lund-Mackay Computed Tomography; SNOT-22: Sinonasal Outcome Test; LK: LundKennedy; SCT: Sinus Control Test.

\section{Internal consistency}

The item-total correlation for each question ranged from 0.4260.624 before surgery and 0.550-0.737 after surgery (Table 4). Before surgery, the overall Cronbach's a was 0.732 and if any one item was deleted it ranged from 0.609 to 0.735 . After surgery, the overall Cronbach's a was 0.782 and if any one item was deleted it ranged from 0.716-0.750 (Table 4).

\section{Floor and ceiling effects}

Before surgery, 4 patients (6.2\%) achieved the highest or lowest possible score. After surgery 3 patients (5.4\%) achieved the highest or lowest possible score.

\section{Discussion}

This paper demonstrates that the SCT is reliable, internally consistent, and responsive to changes after surgical treatment. The test-retest reliability $(0.782)$ was above threshold for acceptability $(\geq 0.70)$ and similar to values reported for previously establis-

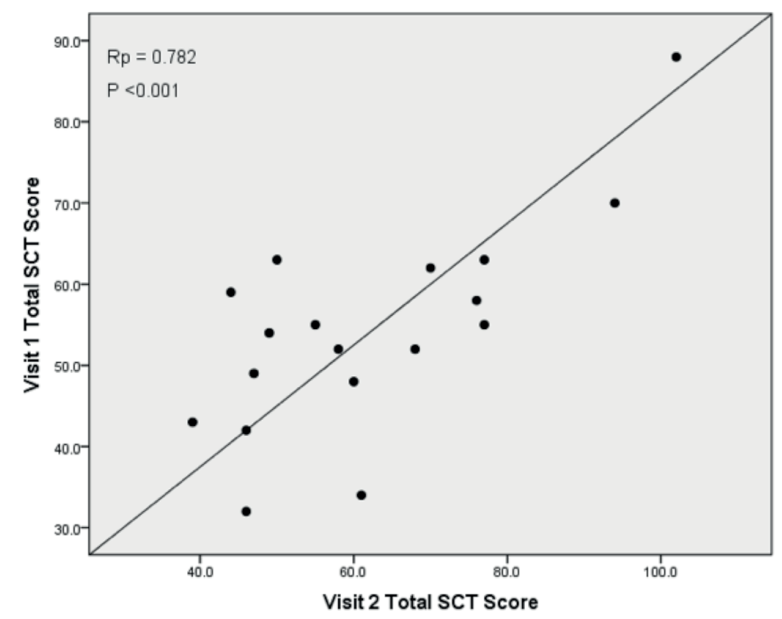

Figure 1. Test-retest reliability.

\section{Sinus Control Test}

1) During the past 2 weeks, how severe was your nasal obstruction?

\begin{tabular}{|c|c|c|c|c|}
\hline None & Minimal & Mild & Moderate & Severe \\
\hline & & & & \\
\hline
\end{tabular}

2) During the past 2 weeks, how severe was your nasal discharge?

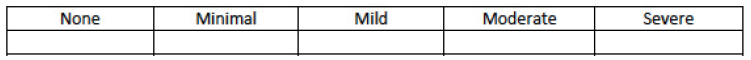

3) How much of the time did your sinus symptoms interfere with getting much done at work or home over the past 2 weeks?

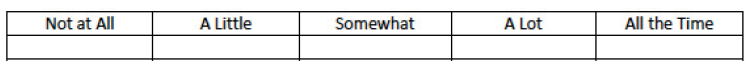

4) Have you had to take a recent treatment of oral steroid or antibiotics in the past 2 weeks for your sinuses?

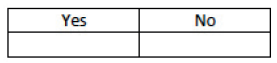

Figure 2. The sinus control test.

hed control tests in asthma and rhinitis ${ }^{(13,14)}$. The reliability of each individual item of the SCT as measured by the ICC was also above the accepted value of 0.40 . For internal consistency, the overall Cronbach's a score was above 0.70 before and after surgery, suggesting the composite SCT consists of individual items measuring highly related concepts. The Cronbach's a values with each item deleted are lower than the overall Cronbach's a, illustrating that the composite questionnaire is more reliable than with any one item deleted. Of note, item 4 showed negligible increase in Cronbach's a when deleted in the pre-surgical group. We also found the SCT to be able to capture the full spectrum of control levels since $<15 \%$ of patients achieved the highest or lowest possible scores before and after surgery. With the addition of this new data, the SCT meets criteria for high quality in terms of PROM development and validation, as described in the systematic review on quality of PROMs in CRS patients by Rudmik et al. ${ }^{(6)}$.

The SCT accounts for sinonasal and systemic symptoms plus 
Table 4. Reliability statistics.

\begin{tabular}{llcccc} 
& & \multicolumn{2}{c}{$\begin{array}{c}\text { Pre-surgical } \mathbf{n = 7 6} \\
\text { ICC } \mathbf{n = 2 2}\end{array}$} & $\begin{array}{l}\text { Post-surgical } \mathbf{n = 6 2} \\
\text { Item-total correlation } \\
\text { Cronbach's a with } \\
\text { item deleted }\end{array}$ & $\begin{array}{c}\text { Item-total correlation } \\
\text { Cronbach's a with } \\
\text { item deleted }\end{array}$ \\
\hline Q1 (nasal obstruction) & 0.518 & 0.586 & 0.681 & 0.737 & 0.716 \\
\hline Q2 (nasal discharge) & 0.765 & 0.624 & 0.609 & 0.599 & 0.727 \\
\hline Q3 (productivity) & 0.868 & 0.560 & 0.650 & 0.550 & 0.750 \\
Q4 (medication use) & 0.724 & 0.426 & 0.735 & 0.599 & 0.726 \\
\hline Overall Cronbach's a & & & $\mathbf{0 . 7 3 2}$ & & $\mathbf{0 . 7 8 2}$ \\
\hline
\end{tabular}

ICC: intra-class correlation coefficient; Q: question

medication use and can help clinicians detect sub-optimally controlled CRS ${ }^{(15)}$. The Asthma Control Test (ACT), a conceptually similar tool for asthmatics, has been shown to help physicians detect uncontrolled asthma and appropriately increase treatment ${ }^{(16)}$. This type of control test is particularly important in primary care settings where physicians continue to see higher volumes of patients, and specialized procedures, such as nasal endoscopy, are not routinely performed. Since primary care physicians are often relied upon to identify disease exacerbations, the SCT in this setting may be especially helpful. Examining the percentages of patients in each SCT category before and after surgery (Table 2), the majority of patients remain in the partially controlled category, even after ESS. This is understandable considering the chronic and relapsing nature of sinusitis but further exemplifies why it is necessary to monitor disease control in this population - administration of the SCT could identify when "partially controlled" patients warrant referral or step-up medication. The SCT provides complementary information to existing quality of life instruments. Change in SCT scores were found to be independent of demographics and co-morbid conditions listed in Table 3. Though age was significantly correlated to change on bivariate analysis, upon controlling for baseline SCT score, it was no longer significant. This illustrates an example of how the SCT could provide additional data on disease state since many PROMs are impacted by co-morbidities like depression and allergy ${ }^{(17-20)}$. Furthermore, pre-surgical SCT scores were correlated with change in SCT whereas pre-surgical SNOT-22 scores were not, suggesting an inherent difference in the elements of disease being measured. Yet, change in SCT was correlated to change in SNOT-22, demonstrating that there is still some overlap in how each is affected by surgery. One can imagine a scenario in which a patient on steroids and antibiotics feels well, elevating quality of life scores, but use of such medications indicates uncontrolled disease, which would be accounted for using the SCT.

Limitations to consider include that this was a single institutional study on patients who presented to a tertiary care rhino- logy clinic, which limits the generalizability of the results to those with milder disease and those living in other geographic locations. We also did not look at the responsiveness of the $\mathrm{SCT}$ in patients before and after medical treatment, which is an area of further study. It is also unknown how the SCT correlates with physician decision-making with regards to treatment. Thus, further study is needed to determine if routine use of the SCT improves treatment outcomes across CRS populations. In addition, the ACT has been cross-culturally validated in several populations world-wide, and similarly, the SCT maybe especially useful in developing countries where resources are limited and patient-reported metrics are often the main methods of guiding therapy.

\section{Conclusion}

The SCT is a brief, validated questionnaire that was developed to help physicians monitor changes in the control of CRS that may warrant change in therapy. It has been shown to be a reliable and responsive tool to assess control of CRS symptoms. Future studies are needed to determine if the SCT leads to improved treatment outcomes when routinely administered in primary care and rhinology clinics.

\section{Authorship contribution}

Concept and design of study and manuscript writing/editing (PK, ZMS, KAS, AS, SMB, RJS); data extraction, analysis, and interpretation (PK, KAS, ZMS, RJS, AS).

\section{Acknowledgements}

Funding: This work was supported by grants from the National Institute on Deafness and Other Communication Disorders (NIDCD), one of the National Institutes of Health, Bethesda, MD (R03 DC013651-01; PI: ZM Soler).

\section{Conflict of interest}

Zachary M. Soler is supported by grants from Entellus, Intersect, and Optinose, none of which are affiliated with this manuscript. 
Dr. Soler is a consultant for Olympus, which is not affiliated with this manuscript. Rodney J. Schlosser is supported by grants from OptiNose, Entellus and IntersectENT, none of which are associated with this manuscript. Dr. Schlosser is also a consultant for
Olympus, Medtronic, Meda, and Arrinex, which are not affiliated with this study. There are no disclosures for Preeti Kohli, Arash Shahangian, Sarfaraz M. Banglawala, or Kristina A. Storck.

\section{References}

1. Soler ZM, Smith TL. Quality of life outcomes after endoscopic sinus surgery: How long is long enough? Otolaryngol - Head Neck Surg 2010;143:621-5.

2. Fokkens WJ, Lund VJ, Mullol J, Bachert C, Alobid I, Baroody F, et al. EPOS 2012: European position paper on rhinosinusitis and nasal polyps 2012. A summary for otorhinolaryngologists. Rhinology 2012; $0: 1-12$.

3. Wechsler ME. Managing asthma in primary care: putting new guideline recommendations into context. Mayo Clin Proc 2009; 84:707-17.

4. Prevention NAE and. Diagnosis and Management. J Allergy Clin Immunol 2007; 120: s94-138.

5. Banglawala SM, Schlosser RJ, Morella K, Chandra R, Khetani J, Poetker DM, et al. Qualitative development of the sinus control test: a survey evaluating sinus symptom control. Int Forum Allergy Rhinol 2015; 6: 491-9.

6. Rudmik L, Hopkins C, Peters A, Smith TL, Schlosser RJ, Soler ZM. Patient-reported outcome measures for adult chronic rhinosinusitis: A systematic review and quality assessment. J Allergy Clin Immunol 2015;136:1532-40e2.

7. Rosenfeld RM, Piccirillo JF, Chandrasekhar SS, et al. Clinical Practice Guideline (Update): Adult Sinusitis. Otolaryngol Head Neck Surg 2015;152:S1-39.

8. Lund VJ, Mackay IS. Staging in rhinosinusitus. Rhinology 1993; 31:183-4.

9. Lund VJ, Kennedy DW. Staging for rhinosinusitis. Otolaryngol Head Neck Surg 1997;

\section{7: S35-40.}

10. Hopkins C, Slack R, Lund V, Brown P, Copley $L$, Browne J. Long-term outcomes from the english national comparative audit of surgery for nasal polyposis and chronic rhinosinusitis. Laryngoscope 2009;1 19:2459-65.

11. Landis JR, Koch GG. The Measurement of Observer Agreement for Categorical Data Biometrics 2008; 33: 159-174.

12. Gliem JA, Gliem RR. Calculating, interpreting, and reporting Cronbach's alpha reliability coefficient for Likert-type scales. Talk presented at: Midwest Research-to-Practice Conference in Adult, Continuing, and Community Education; October 8, 2003; The Ohio State University, Columbus, $\mathrm{OH}$. http://hdl.handle.net/1805/344. Accessed May 1, 2016.

13. Schatz M, Sorkness CA, Li JT, Marcus P, Murray JJ, Nathan RA, et al. Asthma Control Test: Reliability, validity, and responsiveness in patients not previously followed by asthma specialists. J Allergy Clin Immunol 2006;117: 549-56.

14. Meltzer EO, Schatz M, Nathan R, Garris C, Stanford RH, Kosinski M. Reliability, validity, and responsiveness of the Rhinitis Control Assessment Test in patients with rhinitis. J Allergy Clin Immunol 2013; 131: 379-86.

15. Banglawala $S M$, Lawrence LA, Franko-Tobin E, Soler ZM, Schlosser RJ, loannidis J. Recent randomized controlled trials in otolaryngology. Otolaryngol Head Neck Surg 2015; 79 : 418-23.

16. Holt S, Patel M, Montgomery B, Weatherall M, Beasley R. Cohort study of a simple "Step-Up" regimen with the Asthma Control Test. Respirology 2015; 20:504-6.
17. Caten A, Johnson C, Jang D, Gurrola J, Kountakis S. Comorbidities in patients with all-positive symptoms on sinonasal outcomes test quality-of-life instrument. Laryngoscope. 2015; 125: 2648-52.

18. Davis GE, Yueh B, Walker E, Katon W, Koepsell TD, Weymuller EA. Psychiatric distress amplifies symptoms after surgery for chronic rhinosinusitis. Otolaryngol Head Neck Surg 2005; 132:189-96.

19. Brandsted R, Sindwani R. Impact of depression on disease-specific symptoms and quality of life in patients with chronic rhinosinusitis. Am J Rhinol 2007; 21:50-4.

20. Tomljenovic D, Pinter D, Kalogjera L. Perceived stress and severity of chronic rhinosinusitis in allergic and nonallergic patients. Allergy Asthma Proc 2014; 35:398403.

Rodney J. Schlosser, MD

Department of Otolaryngology -

Head and Neck Surgery

Medical University of South Carolina

135 Rutledge Ave

MSC550, Charleston, SC

USA

Tel: +1-843-792-7165

Fax: +1-843-792-0546

E-mail: schlossr@musc.edu 